\title{
ACESSO ANTERIOR PARA PACIENTES COM FRATURAS TRAUMÁTICAS DO TIPO COMPRESSÃO DO SEGMENTO TORACOLOMBAR (T11 A L2) DA COLUNA VERTEBRAL
}

\author{
Asdrubal Falavigna', Orlando Righesso Neto², \\ Maurício Amoretti Polesso3, Paulo Roberto Franceschini ${ }^{3}$
}

\begin{abstract}
RESUMO - Objetivo: Relatar a experiência com o acesso anterior em fraturas traumáticas do tipo compressão no segmento toracolombar (T11 a L2) que foram submetidos à cirurgia pelo acesso anterior. Método: Estudo prospectivo de janeiro de 1994 a janeiro de 2004 envolvendo 32 pacientes. A presença da fusão óssea e do alinhamento foram analisadas 6 e 12 meses após a cirurgia. Resultados: A idade média foi 36,53 anos, sendo 23 do sexo masculino. A vértebra mais atingida foi $L 1(n=12)$. A maioria dos casos que internaram em ASIA/IMSOP C $(n=10)$ e $D(n=13)$ evoluiram para ASIA/IMSOP E. A média da deformidade angular pré-operatória foi $14,9^{\circ} \pm 7,5^{\circ}$, com diferença estatística $(p<0,0001)$ na comparação com o valor encontrado no pós-operatório de 30 dias. Conclusão: A via anterior permite melhor descompressão do canal e uma correção da deformidade angular superior à observada pela via posterior isolada.
\end{abstract}

PALAVRAS-CHAVE: fratura toracolombar, acesso anterior, estabilização.

\begin{abstract}
Anterior approach in patients with traumatic compression fracture type of thoracolumbar spine (T11-L2)

ABSTRACT - Objective: To describe experience with anterior access in compression fractures of thoracolumbar segment (T11 to L2) traumatic fractures that undergone anterior access surgery. Method: A prospective study was conducted between January 1994 and January 2004 with 32 patients. The bone fusion and thoracolumbar alignment were analyzed 6 months and 12 months after the surgery. Results: The average age was 36.53 years old with 23 male patients. The most compromised vertebrae was $L 1(n=12)$. The 23 patients that was ASIA/IMSOP C and ASIA/IMSOP D turned to ASIA/IMSOP E after 1 month $(n=12)$ and 12 months $(n=5)$ of surgery. The preoperative angular deformity average was $14.9^{\circ} \pm 7.5^{\circ}$. Statistical significance was found $(p<0.0001)$ when compared to the 30 days postoperative value. Conclusion: The anterior access permits a better spinal canal decompression and angular deformity correction when compared with the posterior access alone.
\end{abstract}

KEY WORDS: thoracolumbar fracture, anterior access, stabilization.

As lesões agudas da coluna e medula espinhal estão entre as causas mais freqüentes de incapacidade grave e morte após o trauma ${ }^{1}$. Aproximadamente $40,5 \%$ de todas as fraturas de coluna vertebral são encontradas no segmento toracolombar, sendo a fratura do tipo compressão observada em 10 a $20 \%$ dos $\operatorname{casos}^{2-5}$. Em razão desses fatores, a abordagem ântero-lateral foi aplicada com a finalidade de aprimorar a descompressão medular e a reconstrução da coluna anterior, com fusão de menos segmentos vertebrais em comparação com o que ocorre na abordagem posterior ${ }^{6,7}$.
A via de acesso posterior possui como vantagens o conhecimento do acesso da linha média, a ausência de estruturas pulmonares, vasculares e viscerais e a facilidade da revisão cirúrgica ${ }^{6}$. As complicações descritas na literatura são perda do alinhamento, permanência da compressão anterior e falência do sistema de fixação, observadas em $9 \%$ a $54 \%$ dos casos, pela falta de sustentação anterior ${ }^{6-8}$. O acesso anterior é indicado nos pacientes com redução superior a $50 \%$ da altura vertebral e comprometimento do canal vertebral acima de $30 \%$ em T11 e T12, de $40 \%$ em L1 e acima de $50 \%$ em L2, por permitir melhor

${ }^{1}$ Doutor em Neurociências pela Universidade Federal de São Paulo - Escola Paulista de Medicina (UNIFESP-EPM), Professor de Neurocirurgia da Universidade de Caxias do Sul, Caxias do Sul RS, Brasil (UCS); ${ }^{2}$ Ortopedista, Mestre em Ortopedia e Traumatologia pela Faculdade de Ciências Médicas da Santa Casa de São Paulo, São Paulo SP, Brasil; ${ }^{3}$ Acadêmico de Medicina da UCS.

Recebido 23 Fevereiro 2007, recebido na forma final 21 Maio 2007. Aceito 2 Julho 2007. 
descompressão do canal vertebral, sendo observada maior recuperação neurológica quando comparada com o acesso posterior ${ }^{7,9-12}$.

O objetivo do presente estudo é relatar a experiência dos autores com o acesso anterior nas fraturas traumáticas do tipo compressão no segmento toracolombar (T11 a L2).

\section{MÉTODO}

Estudo prospectivo realizado no período de janeiro 1994 a janeiro de 2004 em pacientes com fraturas traumáticas do tipo compressão da transição toracolombar (T11 a L2) que foram submetidos à cirurgia pelo acesso anterior.

Os critérios de inclusão no estudo consideraram os pacientes com fratura e compressão de T11 a L2 associada com retropulsão óssea do corpo vertebral comprometendo mais de $30 \%$ do canal vertebral, preservação de alguma sensibilidade ou motricidade nos membros inferiores, redução superior a $50 \%$ do corpo vertebral e condições clínicas seguras para o procedimento cirúrgico. Foram excluídos os pacientes com lesão medular completa.

O diagnóstico da fratura foi feito por radiologia simples e tomografia computadorizada. Em 17 casos foi possível a realização da ressonância magnética. A deformidade angular foi avaliada através do raio $X$ simples de coluna em perfil, com a medida do ângulo de intersecção dos platôs vertebrais intactos superior e inferior das vértebras adjacentes à fratura.

A classificação de ASIA/IMSOP foi utilizada para avaliação neurológica no momento da internação, 1 mês e 12 meses após a cirurgia ${ }^{13}$.

A via anterior foi realizada através do acesso ântero-lateral, utilizando uma abordagem torácica ou toracoabdominal esquerda, dependendo do nível da fratura e de sua correlação com o diafragma. Houve remoção de costela e secção do diafragma sempre que necessário. $O$ corpo vertebral fraturado foi inteiramente removido e substituído por enxerto ósseo tricortical de ilíaco ou por cilindro de titânio preenchido por osso. Em alguns casos, as vértebras adjacentes foram fixadas com placa e parafusos. Foi necessário a fixação posterior nos casos de instabilidade severa ou quando não foi utilizado material de síntese na via anterior, somente descompressão e colocação do enxerto tricortical de ilíaco.

A via posterior foi realizada através de incisão mediana com exposição de, no máximo, 6 vértebras. Através de fios de cerclagem, parafusos pediculares ou ganchos foram fixados dois ou três níveis acima da fratura e dois níveis abaixo. O enxerto ósseo, retirado do ilíaco, foi colocado nas facetas articulares e nas lâminas previamente driladas. Foi prescrito o uso de colete de polipropileno por 60 dias.

$\mathrm{O}$ alinhamento toracolombar foi analisado no pré-operatório e 1 e 12 meses após a cirurgia através de radiologia simples.

A análise estatística foi realizada com o teste t de Student. Significância estatística foi observada com $p<0,05$.

O estudo foi aprovado pela comissão de ética e os pacientes assinaram o consentimento informado para inclusão do estudo e, havendo assim, o comprometimento do retorno para reavaliação neurológica e radiológica.

\section{RESULTADOS}

Foram estudados 32 casos de fraturas traumáticas do tipo compressão da região toracolombar que necessitaram de descompressão anterior e fixação anterior e/ou posterior (Tabela 1).

A idade média dos pacientes foi 36,53 anos, com preponderância do sexo masculino (23 casos). A idade média dos pacientes do sexo masculino foi 37,86 anos, e a dos pertencentes do sexo feminino, de 33,11 anos (Tabela 1).

A principal causa do trauma foi acidente de trânsito com 21 casos, seguido por queda de altura com 11 casos (Tabela 1).

A vértebra L1 foi a mais atingida, 12 casos, seguindo-se T12 com 8 casos, T11 com 5 casos e a L2 com 3 casos. Fraturas múltiplas foram observadas em 4 casos (Tabela 1).

No acesso anterior, foi utilizado enxerto tricortical de ilíaco em 28 casos e, no restante, cilindro de titânio preenchido com enxerto ósseo da vértebra retirada (Figs 1 e 2). A via anterior isolada foi utilizada em 11 casos, sempre com utilização de placa e parafusos. Quando necessário, a fixação posterior, foi feita com amarrilhas sublaminares e hastes de Hartshill
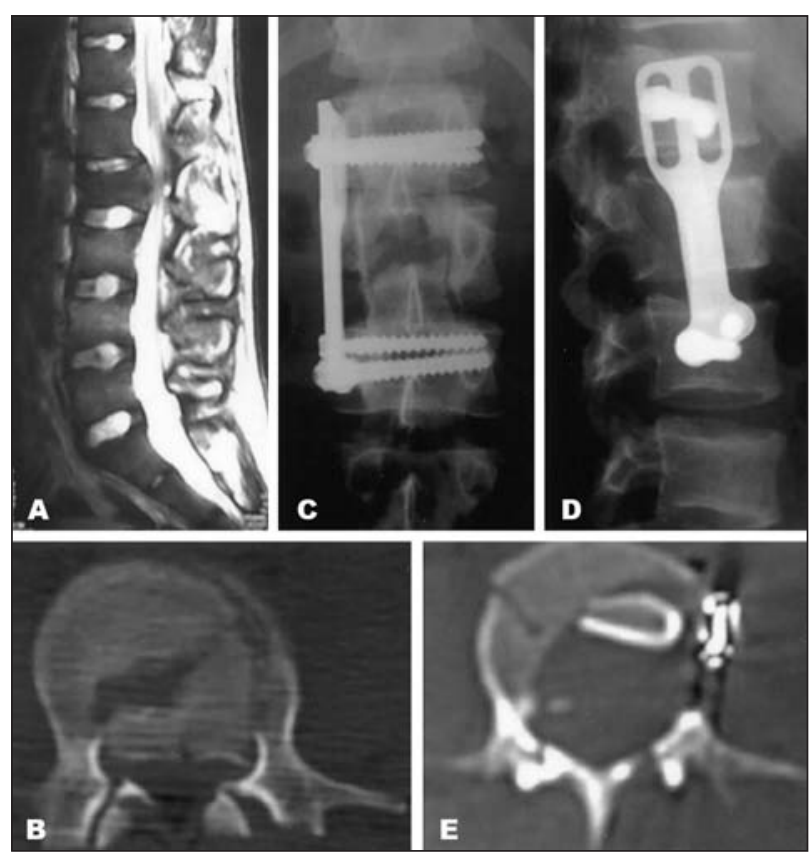

Fig 1. Fratura compressão de $L 2$ (Caso 25). Estudo de imagem pré-operatório com ressonância magnética em corte sagital ( $A$ ) e tomografia computadorizada em corte axial (B). Raio X simples no pós-operatório em visão ântero-posterior (C) e lateral (D) e estudo axial com tomografia computadorizada (E) mostrando a descompressão do canal vertebral e o enxerto de ilíaco. 
Tabela 1. Descrição dos 32 casos de fraturas traumáticas do tipo compressão do segmento toracolombar da coluna vertebral.

\begin{tabular}{|c|c|c|c|c|c|c|}
\hline Caso & Sexo & $\begin{array}{l}\text { Idade } \\
\text { (anos) }\end{array}$ & $\begin{array}{l}\text { Mecanismo } \\
\text { da lesão }\end{array}$ & $\begin{array}{c}\text { Nível } \\
\text { da lesão }\end{array}$ & $\begin{array}{l}\text { Escala ASIA/IMSOP } \\
\text { admissão/6m/12m }\end{array}$ & Tipo de cirurgia \\
\hline 1 & M & 33 & Queda & L2 & $\mathrm{D} / \mathrm{E} / \mathrm{E}$ & VA com enxerto + VP Harrington \\
\hline 2 & M & 18 & Ac. trânsito & L1 & $\mathrm{C} / \mathrm{D} / \mathrm{E}$ & VA com enxerto + VP parafusos \\
\hline 3 & M & 30 & Ac. trânsito & T12 e L1 & $\mathrm{D} / \mathrm{E} / \mathrm{E}$ & VA com enxerto + VP Hartshill \\
\hline 4 & M & 49 & Queda & T12 & $D / E / E$ & VA com enxerto + VP Hartshill \\
\hline 5 & $\mathrm{~F}$ & 67 & Queda & L1 & $D / E / E$ & VA com enxerto + VP Hartshill \\
\hline 6 & $\mathrm{~F}$ & 23 & Ac. trânsito & L1 & $\mathrm{D} / \mathrm{E} / \mathrm{E}$ & VA com enxerto + VP Hartshill \\
\hline 7 & $\mathrm{~F}$ & 22 & Ac. trânsito & T12 & C/D/D & VA com enxerto + VP Harrington \\
\hline 8 & M & 21 & Ac. trânsito & L1 & $\mathrm{C} / \mathrm{D} / \mathrm{E}$ & VA com enxerto + VP Harrington \\
\hline 9 & $\mathrm{~F}$ & 25 & Ac. trânsito & T11 & $B / C / D$ & VA com enxerto + VP Harrington \\
\hline 10 & M & 22 & Ac. trânsito & T12 & $C / D / D$ & VA com enxerto + VP Harrington \\
\hline 11 & M & 64 & Queda & T12 e L1 & $\mathrm{B} / \mathrm{C} / \mathrm{C}$ & VA com enxerto + VP Harrington \\
\hline 12 & $\mathrm{~F}$ & 25 & Ac. trânsito & T11 e T12 & $\mathrm{C} / \mathrm{D} / \mathrm{E}$ & VA com enxerto, placa/parafusos \\
\hline 13 & M & 21 & Ac. trânsito & L1 & $D / E / E$ & VA com enxerto, placa/parafusos \\
\hline 14 & M & 40 & Queda & T12 & $\mathrm{B} / \mathrm{C} / \mathrm{C}$ & VA com enxerto, placa/parafusos \\
\hline 15 & M & 25 & Ac. trânsito & L1 & $\mathrm{D} / \mathrm{E} / \mathrm{E}$ & VA com enxerto, placa/parafusos \\
\hline 16 & M & 65 & Queda & T12 & $D / E / E$ & VA com enxerto + VP Harrington \\
\hline 17 & $\mathrm{~F}$ & 28 & Ac. trânsito & L1 & $\mathrm{D} / \mathrm{E} / \mathrm{E}$ & VA com enxerto, placa/parafusos \\
\hline 18 & M & 24 & Queda & $\mathrm{T} 12$ & $D / D / E$ & VA com enxerto, placa/parafusos \\
\hline 19 & M & 50 & Ac. trânsito & L1 & C/C/D & VA com enxerto, placa/parafusos \\
\hline 20 & $\mathrm{~F}$ & 40 & Queda & L1 & $D / E / E$ & VA com enxerto + VP com ganchos \\
\hline 21 & M & 25 & Ac. trânsito & T11 & $\mathrm{B} / \mathrm{C} / \mathrm{C}$ & VA com enxerto + VP Hartshill \\
\hline 22 & M & 43 & Queda & T11 & $\mathrm{B} / \mathrm{B} / \mathrm{C}$ & VA com enxerto + VP Hartshill \\
\hline 23 & M & 30 & Ac. trânsito & T12 & C/C/D & VA com enxerto + VP com ganchos \\
\hline 24 & $\mathrm{~F}$ & 28 & Ac. trânsito & L1 & $D / E / E$ & VA com enxerto, placa/parafusos \\
\hline $\begin{array}{c}25 \\
(\text { Fig 1) }\end{array}$ & M & 21 & Queda & L2 & $\mathrm{E} / \mathrm{E} / \mathrm{E}$ & VA com enxerto, placa/parafusos \\
\hline 26 & M & 26 & Ac. trânsito & T12 e L1 & $C / C / D$ & $\begin{array}{l}\text { VA com enxerto, placa/parafusos + } \\
\text { VP parafusos pediculares }\end{array}$ \\
\hline 27 & $\mathrm{~F}$ & 40 & Ac. trânsito & T11 & $\mathrm{C} / \mathrm{D} / \mathrm{E}$ & $\begin{array}{l}\text { VA com cilindro, placa/parafusos + } \\
\text { VP parafusos pediculares }\end{array}$ \\
\hline 28 & M & 63 & Ac. trânsito & L1 & $E / E / E$ & VA com cilindro, placa/parafusos \\
\hline 29 & M & 51 & Ac. trânsito & T12 & $\mathrm{B} / \mathrm{C} / \mathrm{C}$ & $\begin{array}{l}\text { VA com enxerto, placa/parafusos + } \\
\text { VP parafusos pediculares }\end{array}$ \\
\hline $\begin{array}{c}30 \\
(\text { Fig 2) }\end{array}$ & M & 53 & Queda & L2 & $\mathrm{D} / \mathrm{E} / \mathrm{E}$ & $\begin{array}{l}\text { VA com cilindro, placa/parafusos + } \\
\text { VP parafusos pediculares }\end{array}$ \\
\hline 31 & M & 63 & Ac. trânsito & T11 & $C / D / D$ & VA com cilindro, placa/parafusos \\
\hline 32 & M & 34 & Ac. trânsito & L1 & $B / C / D$ & $\begin{array}{l}\text { VA com enxerto, placa/parafusos + } \\
\text { VP parafusos pediculares }\end{array}$ \\
\hline
\end{tabular}

$(n=6)$, hastes de Harrington $(n=7)$, parafusos pediculares $(n=6)$ e ganchos $(n=2)$ (Tabela 1 ).

Houve melhora neurológica significativa com o procedimento cirúrgico, não sendo observada qualquer piora durante o seguimento de 12 meses. Os pacientes classificados como ASIA/IMSOP B na admissão ( $n=7)$, evoluíram para ASIA/IMSOP C no período de observação de 1 mês $(n=6)$ e de 12 meses $(n=1)$. A maioria dos 23 casos que internaram em ASIA/IMSOP C $(n=10)$ e $D(n=13)$, evoluiram para ASIA/IMSOP E passados 1 mês $(n=12)$ e 12 meses $(n=5)$ da cirurgia (Tabela 2 ).

A média da deformidade angular na internação foi 14,9 graus com variação de 7,5 . Houve diferença 
Tabela 2. Avaliação neurológica dos pacientes na internação hospitalar segundo a escala de ASIAIIMSOP e sua evolução no período de observação de 1 e 12 meses.

\begin{tabular}{|c|c|c|c|c|c|}
\hline $\begin{array}{c}\text { Escala de } \\
\text { ASIA/IMSOP }\end{array}$ & Admissão & & 1 Mês & & 12 Meses \\
\hline$A$ & 0 & & 0 & & 0 \\
\hline B & 7 & $\begin{array}{l}1 \\
6 \\
\end{array}$ & $\rightarrow 1$ & $\begin{array}{r}1 \\
\end{array}$ & 0 \\
\hline$C$ & 10 & $\begin{array}{r}3 \\
7 \\
\end{array}$ & $\rightarrow 9$ & $\begin{array}{r}4 \\
5 \\
\end{array}$ & $\rightarrow 5$ \\
\hline D & 13 & $\begin{array}{c}1 \\
12 \\
\end{array}$ & $\rightarrow 8$ & $\begin{array}{r}3 \\
5 \\
\end{array}$ & $\rightarrow 8$ \\
\hline $\mathrm{E}$ & 2 & & -14 & 14 & $\rightarrow 19$ \\
\hline
\end{tabular}

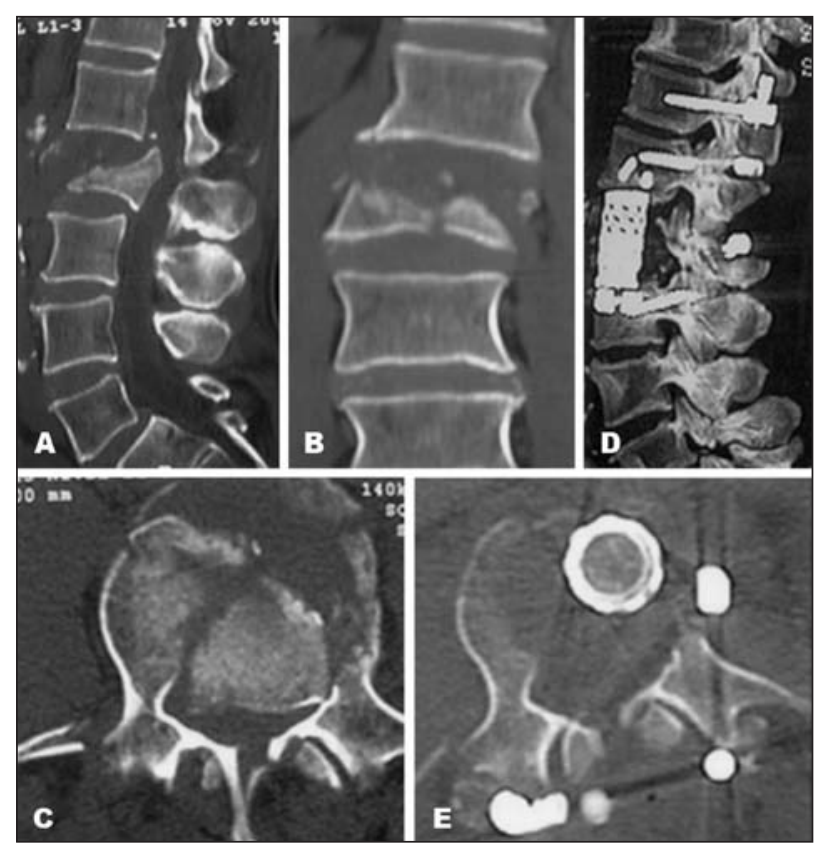

Fig 2. Fratura compressão de L2 (Caso 30). Estudo de imagem pré-operatório com reconstrução tomográfica em vista lateral (A), ântero-posterior (B) e axial (C). Reconstrução tomográfica no pós-operatório em vista lateral (D) e axial (E) mostrando a descompressão do canal vertebral.

estatística $(p<0,0001)$ na comparação com o valor encontrado no pós-operatório de 30 dias, que foi de 3,9 graus com variação de 8,1 . Não foi encontrada significância estatística $(p=0,3046)$, na comparação com o valor passados 12 meses da cirurgia, o qual foi 5,6 graus com variação de 6,9.

Como complicações houve uma violação da cortical do canal vertebral pelo parafuso da placa anterior que necessitou de reposicionamento, dor crônica intercostal em 1 caso, uma hérnia incisional da parede abdominal que necessitou colocação de tela de Marly, 1 caso com trombose venosa profunda e 3 casos de infecção respiratória.

\section{DISCUSSÃO}

Apesar das fraturas toracolombares serem freqüentes, seu tratamento ideal, seja ele conservador ou cirúrgico, permanece controverso ${ }^{11,14-17}$. A cirurgia de descompressão nervosa, alinhamento e fixação da coluna, seja pela via anterior ou posterior, é sugerida na presença de um dos fatores abaixo: distúrbio neurológico progressivo, lesão ligamentar posterior, cifose acima de 20 graus, redução superior a $50 \%$ da altura vertebral e comprometimento do canal vertebral acima de $30 \%$ em T11 e T12, de 40\% em L1 e acima de $50 \%$ em $\mathrm{L}^{7}$. Existe assim, uma variedade de opções cirúrgicas que incluem a redução e descompressão pela via posterior por métodos diretos e indiretos (ligamentotaxia), a descompressão pela via anterior e os acessos ântero-posteriores combinados $^{11,14-17}$.

Esses et al. ${ }^{4}$ realizaram estudo comparativo prospectivo randomizado utilizando o acesso anterior ou posterior em 40 pacientes em um seguimento de 20 meses, analisando a evolução radiológica e clínica. O contorno sagital foi restaurado de maneira similar nos dois acessos, havendo, na via anterior, perda de fixação em 2 casos e maior perda sangüínea. No presente estudo, houve redução significativa da deformidade angular com a via de acesso anterior sem perda do alinhamento sagital passados 12 meses. A manutenção do alinhamento pode ser resultado da cirurgia combinada nos casos de instabilidade severa. Haher et al. ${ }^{18}$ demonstraram em laboratório que as fraturas do tipo compressão deslocam posteriormente o eixo de rotação, sendo este restabelecido com o uso da instrumentação anterior. Kirkpatrick et al. ${ }^{19}$ descreveram uma série de 20 pacientes tratados com o dispositivo Kaneda com $95 \%$ de consolidação óssea. Similarmente, Ghanayem e Zdeblick ${ }^{9}$ relataram uma pequena série de 12 pacientes tratados com ins- 
trumentação anterior para fraturas toracolombares do tipo compressão, sendo que em 11 casos houve excelente resultado funcional associado a uma fusão sólida. Na presente série, não foi observada pseudoartrose e movimentação do enxerto ósseo tricortical de ilíaco ou do cilindro de titânio.

Kaneda et al. ${ }^{14}$ obtiveram um índice de $6 \%$ de complicações com o acesso anterior isolado no manejo de fraturas toracolombares. Neste estudo, houve $21,8 \%$ de complicações, sendo 3 casos de causa cirúrgica direta. Excetuando-se o paciente com dor intercostal crônica em que houve resolução dos sintomas com o tratamento medicamentoso, nos outros 2 casos foi necessário nova intervenção, seja para reposicionamento do parafuso da placa anterior ou fechamento da hérnia incisional abdominal. As outras complicações foram de ordem clínica, trombose venosa profunda $(n=1)$ e infecção respiratória $(n=3)$, sendo observadas nos pacientes de maior dificuldade de motricidade e, por isso, maior tempo de repouso.

Wilson et al. ${ }^{20}$ demonstraram os resultados de 29 pacientes com fraturas toracolombares instáveis no seguimento médio de 20 meses. Os pacientes foram submetidos à corpectomia com colocação de enxerto ósseo e fixação anterior. Evidências radiológicas de consolidação óssea foram demonstradas em todos pacientes. Não foi observada deterioração neurológica pela cirurgia nem complicação tardia relacionada com o sistema de fixação. A fixação posterior foi combinada em 1 caso pela perda da estabilização anterior devido à lesão ligamentar. Parker et al. ${ }^{8}$ estudaram 56 pacientes com fraturas toracolombares, para os quais selecionaram a via de abordagem cirúrgica de acordo com o grau de cominuição, indicando para os casos com menor grau (3-6) a abordagem posterior e para os com maior grau (superior a seis) a abordagem anterior. No presente estudo, a abordagem anterior foi indicada na presença de compressão superior a $30 \%$ do canal vertebral ou redução acima de $50 \%$ do corpo vertebral.

Sasso et al. ${ }^{21}$ analisaram a eficácia da descompressão e reconstrução anterior de fraturas toracolombares instáveis em 40 pacientes, dos quais 37 (95\%) obtiveram uma artrodese estável e $3(8 \%)$ necessitaram complementação da fixação pela via posterior. No presente trabalho, os autores indicaram a complementação posterior em todos os casos em que na via anterior não foi utilizado sistema de fixação interna, somente enxerto tricortical de ilíaco, e em 5 dos 15 casos em que foi utilizada na via anterior a fixação com placa e parafusos.
Wood et al. ${ }^{6}$ realizaram estudo comparativo utilizando a abordagem anterior ou posterior nos pacientes com fraturas toracolombares estáveis sem déficit neurológico, analisando as diferenças radiográficas, clínicas e os resultados funcionais. Os autores concluíram que, embora os resultados com os pacientes fossem similares, a fusão e a instrumentação anterior tendem a apresentar menos complicações ou necessidade de cirurgias adicionais.

Oskouian et al..$^{22}$ revisaram retrospectivamente, de 1997 a 2006 o tratamento cirúrgico pela via anterior em 60 pacientes com fraturas toracolombares instáveis. A média de comprometimento do canal espinhal medido por tomografia computadorizada foi $48,3 \%$, e a de perda de altura vertebral foi $39,4 \%$. A média pré-operatória da deformidade cifótica de $14,9^{\circ}$ chegou a $4,6^{\circ}$ no último acompanhamento. Não houve complicações vasculares nem deterioração neurológica nos casos estudados. Os resultados do presente estudo também demonstram que a redução da deformidade angular é facilmente obtida com a via anterior e que não existe perda da mesma com o passar do tempo.

Sasso et al. ${ }^{23}$ avaliaram 53 pacientes com fraturas toracolombares instáveis. Em 40 deles foi realizado um acesso anterior único e, em 13, um acesso posterior único. Nestes últimos não houve falha na instrumentação, no entanto a perda de correção do plano sagital alcançou uma média de $8,1^{\circ}$ enquanto no grupo dos 40 pacientes ocorreu média de aumento de $1,8^{\circ}$ na cifose. Hitchon et al. ${ }^{24}$ estudaram 63 pacientes com fraturas toracolombares do tipo explosão de T11-L2 em 45 homens e em 18 mulheres. A instrumentação posterior foi realizada em 25 pacientes, e o acesso ântero-lateral, em 38. A média de acompanhamento após a alta hospitalar foi 1,8 anos. As médias pré-operatórias da escala de Frankel nos grupos ântero-lateral e posterior foram, respectivamente, de 3,7 e 3,5 ( $p=0,4155)$. As deformidades angulares pré-operatórias medidas nos grupos ânterolateral e posterior foram, respectivamente, de $11,9^{\circ}$ e $4,1^{\circ}(p=0,0007)$. No pós-operatório, a deformidade foi corrigida para $2^{\circ}$ no grupo ântero-lateral e para $3,4^{\circ}$ no posterior $(p=0,565)$. No acompanhamento, os escores de Frankel melhoraram para 4,2 e 4 nos grupos ântero-lateral e posterior respectivamente $(p=0,461)$. No último acompanhamento, a deformidade angular progrediu para 4,5 no grupo ânterolateral e para 9,8 no grupo posterior $(p=0,024)$. Os autores demonstram que a deformidade angular é melhor corrigida e mantida quando a abordagem anterior é utilizada. 
Cruz $^{25}$ analisou retrospectivamente 27 pacientes com fraturas torácicas e lombares, no período de 2000 a 2003. Foram avaliados radiograficamente no pré-operatório, no pós-operatório e no final do seguimento. A maior parte das fraturas foi na região toracolombar $(70,3 \%)$ com $51,8 \%$ do tipo compressão e $11,1 \%$ do tipo distração. Não houve significância estatística entre as angulações das vias anterior e posterior.

Baseados nos resultados obtidos pode-se concluir que a via anterior permite uma descompressão adequada do canal vertebral e correção da deformidade angular sem perdas significativa da correção passados 12 meses da cirurgia.

\section{REFERÊNCIAS}

1. Bohlman HH. Acute fractures and dislocations of the cervical spine: an analysis of three hundred hospitalized patients and review of the literature. J Bone Joint Surg Am 1979;61:1119-1142.

2. Fessler RG, Masson RL. Management of thoracic fractures. In Menezes AH, Sonntag VKH (Eds). Principles of spinal surgery. New York: McGraw-Hill, 1995:899-918.

3. Kraemer WJ, Schemitsch EH, Lever J, McBroom RJ, McKee MD, Waddell JP. Functional outcome of thoracolumbar burst fractures without neurological deficit. J Orthop Trauma 1996;10:541-544.

4. Esses SI, Botsford DJ, Kostuik JP. Evaluation of surgical treatment for burst fractures. Spine 1990;15:667-673.

5. Muller U, Berlemann U, Sledge J, Schwarzenbach O. Treatment of thoracolumbar burst fractures without neurologic deficit by indirect reduction and posterior instrumentation: bisegmental stabilization with monosegmental fusion. Eur Spine J 1999;8:284-289.

6. Wood KB, Bohn D, Mehbod A. Anterior versus posterior treatment of stable thoracolumbar burst fractures without neurologic deficit: a prospective, randomized study. J Spinal Disord Tech 2005;18(Suppl):S15-S23.

7. Mariotti AJ, Diwan AD. Current concepts in anterior surgery for thoracolumbar trauma. Orthop Clin N Am 2002;33:403-412.

8. Parker JW, Lane JR, Karaikovic EE, Gaines RW. Successful short-segment instrumentation and fusion for thoracolumbar spine fractures: a consecutive 41/2-year series. Spine 2000;25:1157-1170.

9. Ghanayem AJ, Zdeblick TA. Anterior instrumentation in the management of thoracolumbar burst fractures. Clin Orthop Relat Res 1997:89-100.
10. Kostuik JP. Anterior fixation for fractures of the thoracic and lumbar spine with or without neurologic involvement. Clin Orthop Relat Res 1984:103-115.

11. McAfee PC, Bohlman HH, Yuan HA. Anterior decompression of traumatic thoracolumbar fractures with incomplete neurological deficit using a retroperitoneal approach. J Bone Joint Surg Am 1985;67:89-104.

12. Bradford DS, McBride GG. Surgical management of thoracolumbar spine fractures with incomplete neurologic deficits. Clin Orthop Relat Res 1987:201-216.

13. International Standards for Neurological and Functional Classification of Spinal Cord Injury. In: International Medical Society of Paraplegia ASIA/IMSOP 1992: American Spinal Injury Association.

14. Kaneda K, Taneichi H, Abumi K, Hashimoto T, Satoh S, Fujiya M. Anterior decompression and stabilization with the Kaneda device for thoracolumbar burst fractures associated with neurological deficits. J Bone Joint Surg Am 1997;79:69-83.

15. Dimar JR 2nd, Wilde PH, Glassman SD, Puno RM, Johnson JR. Thoracolumbar burst fractures treated with combined anterior and posterior surgery. Am J Orthop 1996;25:159-165.

16. Kaneda K, Abumi K, Fujiya M. Burst fractures with neurologic deficits of the thoracolumbar-lumbar spine: results of anterior decompression and stabilization with anterior instrumentation. Spine 1984;9:788-795.

17. Schnee CL, Ansell LV. Selection criteria and outcome of operative approaches for thoracolumbar burst fractures with and without neurological deficit. J Neurosurg 1997;86:48-55.

18. Haher TR, Bergman M, O'Brien M, et al. The effect of the three columns of the spine on the instantaneous axis of rotation in flexion and extension. Spine 1991;16(Suppl):S312-S318.

19. Kirkpatrick JS, Wilber RG, Likavec M, Emery SE, Ghanayem A. Anterior stabilization of thoracolumbar burst fractures using the Kaneda device: a preliminary report. Orthopedics 1995;18:673-678.

20. Wilson JA, Bowen S, Branch CL, Meredith JW. Review of 31 cases of anterior thoracolumbar fixation with the anterior thoracolumbar locking plate system. Neurosurg Focus 1999;7.

21. Sasso RC, Best NM, Reilly TM, McGuire RA, Jr. Anterior-only stabilization of three-column thoracolumbar injuries. J Spinal Disord Tech 2005;18(Suppl):S7-S14.

22. Oskouian RJ Jr., Shaffrey CI, Whitehill R, et al. Anterior stabilization of three-column thoracolumbar spinal trauma. J Neurosurg Spine 2006;5:18-25.

23. Sasso RC, Renkens K, Hanson D, Reilly T, McGuire RA Jr., Best NM. Unstable thoracolumbar burst fractures: anterior-only versus short-segment posterior fixation. J Spinal Disord Tech 2006;19:242-248.

24. Hitchon PW, Torner J, Eichholz KM, Beeler SN. Comparison of anterolateral and posterior approaches in the management of thoracolumbar burst fractures. J Neurosurg Spine 2006;5:117-125.

25. Cruz CP. Anterior approach versus posterior approach in cirurgical treatment of thoracic and lumbar fractures. Coluna 2006;5:72-76. 